\title{
Vector Analysis and Process Combination in Motion Perception
}

\author{
Hans Wallach \\ Swarthmore College
}

\author{
Robert Becklen \\ Sarah Lawrence College
}

\author{
Donna Nitzberg \\ Swarthmore College
}

\begin{abstract}
Experiments are reported supporting an altered explanation of the vector analysis that occurs in certain motion displays discovered by Johansson (1950). What seemed the result of a perceptual vector analysis is ascribed to the outcome of two different, independent stimulus conditions to which such displays can give rise because of external vector analysis. The different stimulus conditions are configurational change on the one hand and one of the subject-relative stimulus conditions on the other. In two of Johansson's displays, conditions for configurational change were altered by adding stationary reference points in the surround of the displays. Veridical perception of the displays resulted in a majority of instances. We also found that the different motions that result from configurational change and from subject-relative stimulation may combine to form unitary perceived motions and that this happens quite frequently under some conditions.
\end{abstract}

Four conditions of stimulation mediate visual motion perception, three of which are important here. Two represent the changing visual direction of the moving object relative to the observer's eyes. They are the eye movements of ocular pursuit that take place when the eyes track the moving object and the displacement of the image of the moving object on the retinas when the eyes are fixed on a stationary point. Because they register an object's motion relative to the observer, these two stimulus conditions are called subject-relative. The third stimulus condition, configurational change, represents the displacement of the moving object relative to its stationary background or to stationary objects in its surround. By its very nature, configurational change can function also nonveridically; the same conditions that mediate real motion can cause perceived motion when the object is stationary and its background moves. The nonveridical perceived motion of the object that results is called induced motion. Because the subject-relative

This work was supported by Grant 11089 from the National Institute of Mental Health to Swarthmore College, Hans Wallach, principal investigator.

Requests for reprints should be sent to Hans Wallach, Department of Psychology, Swarthmore College, Swarthmore, Pennsylvania 19081. stimulus conditions represent the object correctly as stationary, induced motion is usually the result of a cue conflict. ${ }^{1}$ In the perception of real motion, on the other hand, ocular pursuit or image displacement provides the same information about the objective motion as does configurational change. For that reason, investigations of configurational change usually make use of the induction condition.

Using the conflict between configurational change and the subject-relative conditions of stimulation that occurs in induced motion, Wallach, O'Leary, and McMahon (1982) compared the effectiveness of three stimulus conditions and found that configurational change and image displacement were about equally effective but that configurational change proved more potent than ocular pursuit when the two were in conflict with each other. Then induced motion was fully effective, that is, its extent was equal to the extent of the motion of its background.

That the three conditions of stimulation evoke different processes is obvious: One registers eye movements, another involves changes in retinal location, and the third is a matter of form perception. There is evidence

\footnotetext{
${ }^{1}$ An exception is the condition where the velocity of the backgroung motion is below the subject-relative threshold.
} 
that the results of two different processes can combine in such a way that a single motion is perceived. The display used by Wallach et al. (1982) affords an example of this. A light dot, which moved up and down, was surrounded by a pattern of vertical lines, which moved alternately to the left and to the right, in phase with the dot's motion. The displacement between the lines and the dot caused the dot's horizontal induced motion. Because the dot, moving vertically on the vertical line pattern, encountered no landmarks, its motion was given only subject-relatively. Two different stimulus conditions caused the dot to move; ocular pursuit or image displacement mediated its vertical motion, while at the same time configurational change caused it to move horizontally. In the end, the two motion processes combined, and the dot was seen to move on an oblique path, the resultant of two motion processes-a vertical and a horizontal one-which were caused by different stimulus conditions. Such an event we call process combination.

Different motion processes involving the same object, however, do not always combine to result in a single motion path. On the contrary, single motion paths may give rise to two perceived motions that have been considered kinematic components of the object's given path, the apparent result of a vector analysis. The relation between this vector analysis and the existence of different stimulus conditions for motion is the issue of the following discussion.

Instances of such vector analysis were described by Rubin (1927) and by Duncker (1929), but we owe a systematic treatment and the discovery of further instances to Johansson (1950). In the simplest of these instances, two dots move back and forth on different paths, one vertical and the other horizontal (Johansson's Experiment 20; see Figure 1, Panels A and B). They move at the same rate and reverse their motions simultaneously. These crossing paths, however, are not perceived unless one of the dots is tracked. Instead, each dot undergoes two simultaneous motions. They move colinearly toward and past each other and back on paths that, for example, are oriented, northwest to southeast, and, at the same time, they move together back and forth at right angles to the colinear

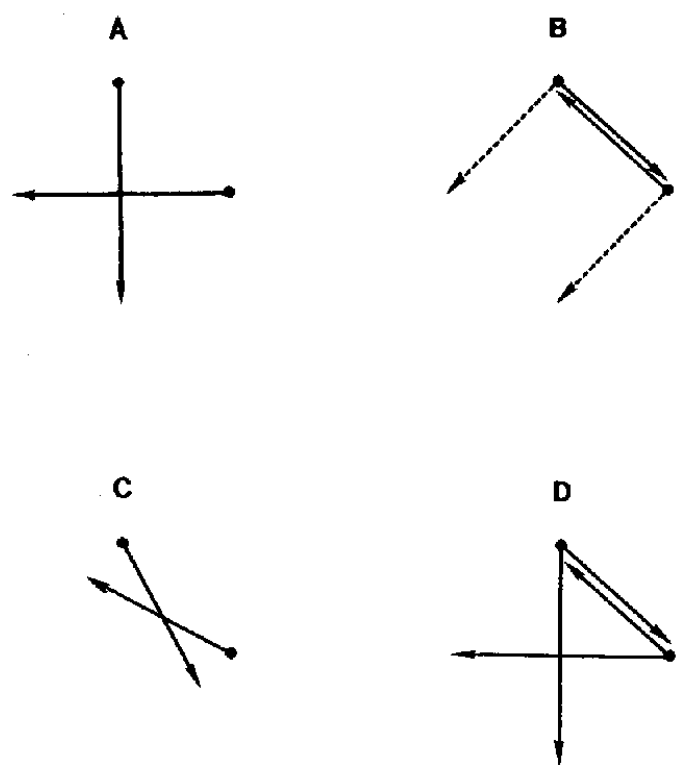

Figure 1. Panel A shows the given motion pattern of Johansson's crossing paths display. Panel B shows the perceived motions that have previously been described. (Motions in the directions of the dashed arrows are sometimes not reported.) Panel $\mathrm{C}$ shows the perceived motion paths we ascribe to process combination, and Panel D the two stimulus conditions that are effective when the combined motions shown in Panel $\mathrm{C}$ are perceived.

paths, namely between northeast and southwest. For each dot, the kinematic resultant of its simultaneous motions is in agreement with its actual path. Therefore, the two simultaneously perceived motions have been regarded as the outcome of a vector analysis.

At the North Atlantic Treaty Organization conference entitled "Symposium on the Study of Motion Perception, Recent Development and Applications," held in 1980 in Velthoven, the Netherlands, Wallach read a paper that was subsequently published by Wertheim, Wagenaar, and Leibowitz (Wallach, 1982). In this paper, he proposed a different explanation of the observations that have been ascribed to vector analysis.

It assumes that the spots that make up the moving pattern form a group prior to motion perception, and that the displacement of the group as a whole, which is subject-relatively given, gives rise to one of the perceived motions. The other perceived motion results from the relative displacements within the group, which are given as configurational change. In the present example the two spots as a group are objectively displaced between 
NE and SW. That displacement gives rise to one of the perceived motions. The displacement of the spots relative to each other is on an oblique line connecting them. It is given as configurational change and gives rise to the other perceived motions. The displacement of the group as a whole, on the other hand, is only subject-relatively given. Thus, different kinds of motion processes give rise to the component motions that are perceived. Thus . . different motion vectors are perceived because different kinds of processes cause them; the two component processes come into play for different reasons (Wallach, 1982, p. 8).

In other words, we are not dealing with a special process where the vertical motion of one dot and the horizontal motion of the other dot are each replaced by two motions, which are paired off with each other, two being colinear and two having the same direction. Rather, inherent in the given motion pattern are two stimulus conditions that cause different motion processes from the outsetthe configurational change between the dots and the subject-relative stimulations that they also provide. To be sure, vector analysis makes the two stimulus conditions possible, but it is a vector analysis of the physical motions that gives rise to the two stimulus conditions. (We are referring here to the simple fact that, in the physical world, two or more arbitrarily selected component motions of which the given motion is the resultant are just as real as the given motion. Because stimuli are part of the physical world, stimulation will just as readily be caused by component motions as by the given resultant motion.) Why the independent motion processes that result from the two stimulus conditions do not always combine in the end as happens in connection with the type of display used by Wallach et al. (1982)-it turned out that they sometimes do-is one of the topics of this article.

This interpretation can be applied to other displays discovered by Johansson, one of which will be discussed in detail below. It also fits the rolling-wheel displays where vector analysis seemed to take place, except that here still another stimulus condition, orientation change, plays the role that configurational change plays in the Johansson displays. ${ }^{2}$

This interpretation of the way in which rolling wheel and Johansson displays are perceived has the advantage of simplicity: The perceived component motions result from different stimulus conditions, which give rise to different perceptual processes that end in two simultaneous perceived motions. That a single objective motion can produce two simultaneous perceived motions has been known since Duncker (1929) explained induced motion. In induced motion, too, a given single motion produces two perceived motions, the motion of the surround via subject-relative stimulation and the motion of the surrounded object through objectrelative stimulation that we now call configurational change. ${ }^{3}$ In instances of vector analysis, two perceived motions also result from the same stimuli and from the same independent motion processes that operate when induced motion displays are observed, except that one object undergoes the two perceived motions. This explanation of vector analysis, thus, is based on the most elementary facts of motion perception, namely, that several stimuli mediate it. Although this explanation hardly needs support, we thought that it might be helpful to demonstrate the role that configurational change plays here. We did this by adding configurational elements without altering the given dot motions. In the crossing paths display, only one configurational change takes place: The two dots change their distance from each other. Adding stationary dots means adding further configurational changes, which take place between them and the moving dots. They may cause the distance change between the moving dots to lose its dominance, and that is what happens. Under these conditions, the distance change between the moving dots no longer gives rise to their colinear paths. Rather, because the objective vertical and horizontal paths are not given by configurational change also, a change away from the perception of the component motions toward seeing the vertical and the horizontal (VH) motions occurs, as shown by our first experiment.

\footnotetext{
${ }^{2}$ Orientation change cannot be subsumed under configurational change because it takes place in relation to the observer's coordinates. We consider erroneous the first author's argument presented in Wallach (1965) that orientation change is a configurational change.

${ }^{3}$ A subject of debate has been only whether the two perceived motions divide the given relative displacement between each other or whether the sum of their extents can exceed it. (Rock, Auster, Schiffman, \& Wheeler, 1980, vs. Wallach et al., 1982).
} 


\section{Experiment 1}

A crossing paths display was presented either in total darkness or with eight stationary dots surrounding it, and descriptions of the apparent motions were elicited.

\section{Method}

Subjects. Twenty undergraduates served as subjects.

Equipment. The apparatus used by Wallach and Becklen (1983) was modified so that two lanterns each projected a bright dot. One beam was reflected by a mirror that turned on a horizontal shaft and the other beam by a mirror that turned about a vertical shaft in concert with the first mirror. Secondary mirrors reflected the beams toward a large translucent screen $(145 \mathrm{~cm} \times$ $155 \mathrm{~cm}$ ) where the dots came to a focus. The mirror excursions and the projection distances were such that the paths over which the dots shifted in reciprocating motion were of equal length. The dot pattern that surrounded the moving dot display in one of the experimental conditions was projected by a third lantern. The moving dots were $3 \mathrm{~mm}$ in diameter, and their paths were $10 \mathrm{~cm}$ long. Their reciprocating motions were approximately simple harmonic, and the speed was 0.15 cps. Eight stationary dots of $8 \mathrm{~mm}$ diameter, which could be added to the moving dot display, were arranged in a square that measured $12.5 \mathrm{~cm}$ on one side and surrounded the moving dots symmetrically. The subjects, who wore the frames of welder's goggles to make sure that the screen edges could not be seen, observed from a distance of $150 \mathrm{~cm}$.

Procedure. Because we knew that perceiving the component motions is more easily disturbed, the two-dot display was always presented first, and the display with the framework of added stationary dots came second. After observing a display, the subject drew the motion paths he or she had seen on a card and gave an explanatory verbal description. Finally, a forced-choice test was added at the end of the experiment in the case of 15 subjects. After being given descriptions of the two possible motion patterns, the component motions, and the VH motions, the subjects were then asked which description seemed more compelling for each display. They were told that both descriptions were equally "right."

\section{Results}

In the forced-choice test all 15 subjects found the component motion description more compelling in the two-dot display and the description of the $\mathrm{VH}$ motions correct for the frame display, as we expected.

The results of the drawing test were more interesting. In connection with the two-dot display, 9 subjects drew the oblique colinear motion of the dots. (Only 4 of these drew the other motion vector, the one that is perpendicular to the colinear path. That is not unusual; the latter motion is often not reported.) Ten subjects drew oblique paths that deviated from being colinear and crossed each other at an angle of between $30^{\circ}$ and $40^{\circ}$. One subject drew crossing curved lines. In the frame condition, 17 subjects drew a vertical and a horizontal path, and 1 drew curved paths that crossed under $90^{\circ}$. Only 2 subjects made drawings resembling those made in connection with the two-dot display; one drew colinear paths, and the other drew paths that crossed under $40^{\circ}$. Adding the frame thus produced 18 out of 20 drawings that indicated that VH motions were seen, whereas no such drawings were made in connection with the two-dot display.

We propose that the 10 instances of motion paths crossing at angles of $30^{\circ}$ and $40^{\circ}$ that occurred in the two-dot condition are the result of a combination of two motion processes, one that results from the configurational change between the dots and the other from the subject-relatively given vertical and horizontal displacements of the individual dots (Figure 1, Panels C and D). Why that combination does not always take place will be discussed later.

\section{Experiment 2}

The experiment that had been done with the crossing paths display was repeated with another instance of vector analysis that Johansson had discovered (shown in Johansson's Figure 39). In this display two dots undergo different reciprocating motions in concert, one moving up and down on a straight path and the other moving on a circular path. (See the straight and one circular motion in Figure 2, Panel A). A circular motion path can be the kinematic resultant of two straight simple harmonic motions that form a right angle with each other and are combined with a phase shift of $90^{\circ}$. Therefore, a circular path 
can simultaneously give rise to two stimulus conditions, one representing a horizontal and the other a vertical path. This can happen when the circular motion occurs in the context of the motion of another dot that is apt to be a partner in a configurational change and a partner in a group motion. That is the case in the Johansson display, where the straight vertical motion of one dot is next to the circular motion of the other dot and is synchronous with the latter's vertical component. Under these conditions the two dots undergo simultaneous subject-relative displacements up and down, making use of the vertical component of the circular path. At the same time, they undergo a configurational change in which the distance between the dots changes periodically in the horizontal dimension. The two stimulus conditions often give rise to the perceived motions that $\mathrm{Jo}$ -
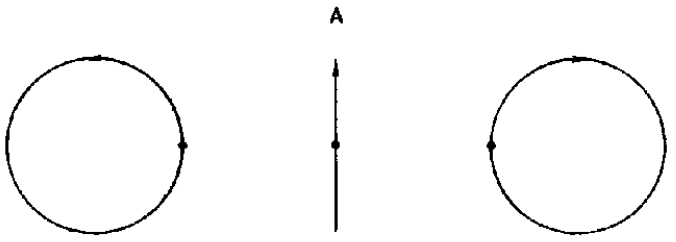

B
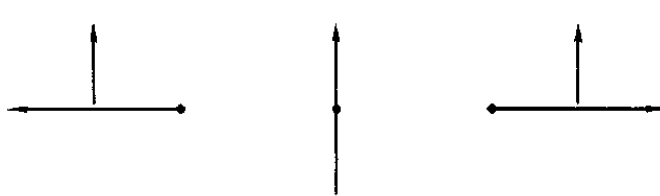

Figure 2. Panel A shows the actual motion paths of the three LEDs in Experiment 2. (The circling light-emitting diodes (LEDs) revolve in opposite directions; the LED in the center moves up and down. The three spots mark simultaneous locations of the LEDs, with the one in the center on the way up at the time.) Panel B shows the perceived motions of the LEDs in accordance with vector analysis and reported by 21 of our subjects when the stationary LEDs were not visible. (The spots mark the same simultaneous locations of the LEDs as in Panel A. The LED in the center is perceived to move up and down, and the lateral LEDs are engaged in two simultaneous motions, one horizontally back and forth and the other up and down. At the time of the locations of the LEDs depicted by the spots, the lateral LEDs move outward and upward.) hansson described: The dots appear to move up and down, and one simultaneously undergoes horizontal motion. For a reason that will be discussed later, these motion patterns are reported more frequently when two circular paths are displayed that are located symmetrically on either side of the vertical path, and that is the form in which we used this kind of display. (See Figure 2, Panels A and B). As was done in connection with the crossing paths display, we added in one condition a frame of stationary dots that provided further configurational changes capable of representing the objective dot motions and hoped thereby to eliminate the dominant role of the distance change between the moving dots that normally caused the horizontal motion of the dots in the perceived motion pattern.

\section{Method}

Suhjects. Thirty-eight undergraduates served as subjects.

Equipment. The three moving dots were 2.1-V light emitting diodes (LEDs) attached to a specially constructed mechanical device. The two LEDs that moved on circles were mounted on pins that were inserted in metal disks. The disks were attached to shafts that turned in horizontal bearings. Sprocket wheels that were mounted on the other end of each shaft and a sprocket belt formed a connection between the shafts, one of which was driven by the slow shaft of a Bodine shunt motor. Metal bars mounted between the disks formed a vertical slot in which a small carriage could move up and down to which the third LED was attached. A horizontal bar with a lengthwise slot was attached to the carriage so that it passed in front of one of the metal disks. The LED carrying pin on that disk passed through this slot and fitted it snugly. Hence the slotted bar picked up the vertical component of the circling motion of the LED and caused the carriage LED to move up and down in concert with the circling LED. All metal surfaces were painted matte black.

The diameter of the circular paths of the outer LEDs was $9 \mathrm{~cm}$, and the excursion of the vertically moving LED was also $9 \mathrm{~cm}$. The distance between the centers of the circular paths was $25 \mathrm{~cm}$, so that the nearest point of each circular path was $8 \mathrm{~cm}$ from the path of the vertically moving LED. Six stationary LEDs were arranged on a frame so that they surrounded the moving display symmetrically. One was located on each side, on a line with the center of the display and $9 \mathrm{~cm}$ from the centers of the circular paths, and one pair was above and a second pair below the display. Their vertical distances were $19 \mathrm{~cm}$, and the distances within the pairs were 20.3 $\mathrm{cm}$. During observations the room was dark. A transparent mirror was placed in front of the display, dimming the 
LEDs and keeping the device on which they were mounted invisible when the room was illuminated. The subject, who sat $4.6 \mathrm{~m}$ from the display, wore goggles with neutral density filters. The LEDs completed a circular path in $1.43 \mathrm{~s}$. These circling LEDs moved in opposite directions and were, at any moment, horizontally aligned with the center LED and equidistant from it.

Procedure. A subject first viewed a moving display in the dark for $20 \mathrm{~s}$. Then a lamp was turned on, and the subject drew the motion paths that he or she had seen and at the same time gave a verbal description. Half the subjects were first shown the moving display without the stationary LEDs and then the display with the stationary LEDs, and the other subjects observed the two displays in the opposite order. After the expected effect of the stationary LEDs had been obtained with most of 22 subjects, their number was diminished from six to four; only the LEDs above and below the moving LEDs were visible in the case of these subjects.

\section{Results}

When the three moving LEDs are given without the stationary LEDs, the configurational change between the horizontal motion components of the circular paths of the outerlight dots and the center dot consists in the outer dots alternately approaching the center dot and receding from it. This configurational change brings about the component motions that are seen when the perceived motion pattern corresponds to Johannson's decription; the outer dots move horizontally, alternately inward and outward. The subjectrelative displacement of the three dots as a group is up and down. It gives rise to the other component motion that is frequently spontaneously reported. As in our previous experiment, process combination may take place. In that case the outer dots appear to move on elliptic paths in horizontal orientation. Finally, the perceived motion may resemble the objective motion paths. In that case, the subject reports circular paths of the outer lights.

Five subjects drew a variety of motion paths that could not be interpreted. The results for the remaining 33 subjects are listed in Table 1. In the condition without the stationary dots, only 4 subjects drew and reported circular paths. The remaining 29 subjects saw the outer dots move according to configurational change, either horizontally or, modified by combination with the motion process produced by subject-relative stimu-
Table 1

Number of Subjects Who Drew the Motion Path of Both or of One Circling Light-Emitting Diode(s) (LEDs) in One of Three Shapes in Two Conditions of Experiments 2 and Experiment 3

\begin{tabular}{|c|c|c|c|}
\hline \multirow[b]{3}{*}{ Path shape } & \multicolumn{3}{|c|}{ Stationary LEDs } \\
\hline & \multicolumn{2}{|c|}{ Experiment 2} & \multirow{2}{*}{$\frac{\text { Experiment } 3}{\text { Absent }}$} \\
\hline & Present & Absent & \\
\hline Circular & 28 & 4 & 4 \\
\hline Linear & 3 & 21 & 7 \\
\hline Elliptic & 2 & 8 & 16 \\
\hline
\end{tabular}

lation, on elliptic paths. With the stationary LEDs added to the display, 28 subjects drew circular outer paths. The other two paths that resulted when configurational change among the moving dots was effective numbered only five. As had happened in Experiment 1, adding a frame of stationary dots strikingly increased the frequency with which the objective motion paths were perceived. When configurational change among the moving dots was effective, subjects almost always reported spontaneously a simultaneous motion of the three dots moving up and down. This motion was large when the circling dots were reported to move horizontally and smaller when process combination occurred and the outer dots moved on elliptic paths. At that point, the third dot moved only as much as the vertical extent of the elliptic paths.

The combination of different motion processes. We turn now to the question why the two motion processses that result from the two stimulation conditions to which the Johansson displays give rise do not always combine. We propose that the same grouping of the two or three dots that causes the parallel components of their subject-relative displacements to be the effective stimulation prevents the subsequent individual processes from combining with the configurational motion processes. (When this combination takes place, the subject-relative motion directions differ from each other, and loss of grouping is implicit.) Grouping is likely to be stronger, and consequently instances of combination should be fewer when the number of dots 
that form the group is larger. This seems to be confirmed when the results of the conditions without the stationary dots of Experiments 1 and 2 are compared. Among the 19 instances in Experiment 1 where the perceived motions did not resemble the objective motion paths, the paths of $10(53 \%)$ crossed at $30^{\circ}-40^{\circ}$ angles, presumably the result of process combination. Here two dots formed the group. Among the corresponding 29 instances of Experiment 2, where three dots formed the group, there were only $8(28 \%)$ instances where the outer dots seemed to move on elliptic paths, which are presumed to result from process combination. This comparison is suggestive but obviously not conclusive. We therefore modified the condition without stationary dots of Experiment 2 by omitting one of the circling LEDs so that only two dots were seen.

\section{Experiment 3}

Forty-four subjects observed the display without the stationary dots, with one of the circling LEDs disconnected. In all other respects, conditions and procedure were the same as in Experiment 2.

Seven subjects drew a variety of motion paths that could not be interpreted, and 10 subjects drew two circles. The latter is the outcome when the circling LED is tracked. ${ }^{4}$ The results for the remaining 27 subjects are listed in the last column of Table 1. As happened in the corresponding condition of Experiment 2, few subjects, namely 4, saw the circling LED move on a circular path, and a group of 23 subjects saw this dot move according to configurational change, either on a linear horizontal path or on an elliptic path. But within this group the results of Experiment 2 and Experiment 3 were quite different. In Experiment 3, a large number of subjects (16) reported the elliptic path, which is presumably the outcome of process combination, and only 7 subjects reported the linear path. This is very different from the results of Experiment 2, where only 8 subjects reported the elliptic path and 21 the linear path. The difference between these results was significant $\chi^{2}(1,52)=7.48, p<$ $.01)$. As expected, diminishing the number of moving dots in the display from three to two, and along with it the grouping strength, resulted in a higher proportion of process combination.

\section{Summary and Discussion}

Because it is the nature of physical motion that any motion can be the resultant of two or more component motions, some motion patterns that consist of more than one moving object can act as two different stimulus conditions and simultaneously evoke two perceived motions that represent components of the given physical motions. This is possible because we are equipped with different sensory responses to motion, which fit different kinematic components of the objective motions. This is our explanation of the pattern of perceived motions that have been considered the result of a psychological vector analysis, either a matter of extracting a common motion components (Johansson, 1950) or of extracting the components that are given as configurational change, with the residual displacements being the other perceived motion (Wallach, 1965). Rather, these motion patterns are an incidental result of our sensory equipment. There is no need for postulating an extraction process.

Our experiments dealt with motion patterns with physical components that were represented to the eyes as configurational change. We demonstrated the crucial role of configurational change by adding stationary dots to the motion displays and thereby altered configurational change without the given motion pattern's being altered. This resulted in changed perceived motions. These results are not immediately compatible with one of the earlier views on vector analysis, namely that it consists in the extraction of the common motion components; there is no reason why the presence of stationary dots should prevent such an extraction. Other kinematic components that are possible in these motion

\footnotetext{
${ }^{4}$ An explanation of this outcome has been presented in Becklen, Wallach, and Nitzberg (1984) in connection with an experiment where subjects were instructed to track the circling LED.
} 
patterns fitted other stimulus conditions, those that represent subject-relative changes, changes between a moving object and the observer. These stimulus conditions evoked processes that often resulted in perceived motions that corresponded to these motion components.

It is known that the processes that are the outcome of configurational change and of one of the subject-relative stimulus conditions can combine and result in a single experienced motion path, and that can also happen in the case of the two motion patterns with which we experimented. In the crossing paths display, the combined motions consisted of motion paths that crossed at $30^{\circ}-40^{\circ}$ angles instead of being colinear, and in the circling dot(s) displays the combined motions consisted of elliptic motion path(s) instead of linear ones. But that does not always happen. In the case of the crossing paths display, half the subjects saw the dot move colinearly, and some of them reported simultaneous second motions: The dots moved together, orthogonally to the colinear paths. In Experiment 2, a large majority of subjects saw the circling dots move in horizontal motions in accordance with the configurational changes in which they partook, and all of these subjects reported that the three dots moved together up and down. In both cases these common motions resulted from the parallel components of the subject-relative displacements of the dots.

Why is it that the combination of the two simultaneous motion processes that the different stimulus conditions evoke often fails to take place? We proposed that the same group formation between the dots that causes their common motions based on the parallel components of their subject-relative displacements prevents process combination, and we supported this view by showing in Experiment 3 that diminishing grouping strength increased the rate at which process combination occurred. Process combination is the reason why the objective motions, which are given subject-relatively as an alternative to the parallel components, are so rarely perceived. The objective motions are given subject-relatively when grouping fails, but in that case process combination takes place.
It seems peculiar that the perceived motion patterns that we ascribe to process combination have not been previously investigated. This is probably due to the scarcity of more detailed investigations of instances of vector analysis. Hochberg and Fallon (1976) were the first to measure the effect of vector analysis. They used a pattern of three linearly moving dots. The apparent motion direction of a dot that we would expect to move in accordance with configurational change was matched with the motion direction of a single dot. Under conditions where, corresponding to complete vector analysis, the motion of the critical dot resulted solely from configurational change and its motion would have had a direction of $90^{\circ}$ and where the objective motion had a direction of $45^{\circ}$, a mean direction of $69.8^{\circ}$ was measured. How much, if any, of the deviation from $90^{\circ}$ resulted from process combination and how much was due to the presence of a stationary spot, which was needed as fixation mark and incidentally provided an additional configurational change, is not clear.

Restle (1979) employed an information processing approach to vector analysis: Where several interpretations of a given pattern of motions are possible, that one is perceived that is the most economical in terms of information load. This approach was remarkably successful in predicting the motions that result from vector analysis. Their information load is lower than that of the objective motions that they replace. The approach, however, fails to predict the result of adding stationary dots in the surround of our motion displays. We found that this addition strongly favors the perception of the objective motions, but the objective motions carry a higher information load than the motions that are seen when the stationary dots are absent. Because it is inconceivable that adding stationary dots can lower the information load of the objective motions, Restle's approach does not fit here.

Considerations of information load were also successful when a pair of objective motions yielded several different motion patterns. In cases of such ambiguity, information loads turned out to be equal. But that does not apply to the motion paths that in our experiments result from process combination. They 
carry higher information loads than the motion patterns with which they occur interchangeably.

Shum and Wolford (1983) experimented with an arrangement of three dot motions, two linear vertical paths flanking an elliptical path, as shown in Figure 14 in Restle (1979). The elliptical path was of constant width, but its height varied in six steps beween 0 and a height/width ratio of 2 . The length of the two vertical paths was always equal to the width of the center path. The subject selected from an array of drawn ellipses the one that fitted the shape of the center path. The results for the six motion paths were pooled and reported in accordance with a formula,

$$
M_{p}=M_{a}-k M_{f},
$$

where $M_{\mathrm{p}}$ is the shape of the perceived path as measured by the height of the selected ellipse, $M_{\mathrm{a}}$ the height of the actual elliptical path, and $M_{\mathrm{f}}$ the length of the actual linear paths. The dependent variable $k$ "specifies the proportion of the common motion that is extracted" (Shum \& Wolford, 1983, p.19) and is a measure of the perceptual vector analysis that is achieved. When $k M_{\mathrm{f}}$ is larger than $M_{\mathrm{a}}, M_{\mathrm{p}}$ becomes negative, and that means that the motion direction of the center dot is reversed. This result was indeed obtained in the case of the flat ellipses. This reversal of the motion direction is a striking fact $^{5}$ and requires an explanation.

Becklen, Wallach, and Nitzberg (1984) have shown that position constancy does not operate when a target moves during a pursuit movement in a direction that forms an angle with the direction of the eye movement. In that case, the target's perceived motion path depends on the path of its image on the retina. This could happen in those arrangements used by Shum and Wolfort where the motion path of the center dot is a flat ellipse if the eyes track the vertically moving dots. Even small vertical eye movements would result in image paths that are different from the objective paths. These image paths would still resemble ellipses, and they would be traversed in reversed direction if the vertical eye movements are larger than the height of the given elliptic path. Very small vertical tracking movements would suffice to cause reversed perceived motion directions in the cases of ellipses with height/width ratios of 0 and .125.

Some of the six experimental conditions that were used by Shum and Wolford may have yielded process combination. Unfortunately, the means for the perceived shapes of the six different motion paths were not reported. The high mean $k$ value that was calculated for the pooled results does not necessarily mean that process combination was rare or absent. The high mean may be an artifact of three very flat ellipses having been used among a total of six, those with height/width ratios of $0, .125$, and .25 , which frequently may have yielded negative values of $M_{\mathrm{p}}$.

In another important investigation by Proffit, Cutting, and Stier (1979), orientation change played the role of configurational change. This investigation will be discussed in a different article, in which experiments with a rolling wheel display will be reported.

Approaches to vector analysis that do not take the basic facts known about motion perception into account are in danger of being of limited value. By contrast, our approach started with the different stimuli that mediate motion perception and result in different perceptual processes that operate, either totally or up to a point, independently of each other. It turned out that these facts predict, without further assumptions, perceived motions that had previously been considered the outcome of the extraction of the common motion components or of the components engaged in configurational change.

\footnotetext{
${ }^{5}$ It had also been sometimes observed by Restle (1979) in the display shown in his Figure 14, where the height of the ellipse was half of the length of the flanking dot paths.
}

\section{References}

Becklen, R., Wallach, H., \& Nitzberg, D. (1984). A limitation of position constancy. Journal of Experimental Psychology: Human Perception and Performance, 10, 713-723.

Duncker, K. (1929). Über inducierte Bewegung [About induced motion]. Psychologische Forschung, 12, 180259.

Hochberg, J., \& Fallon, P. (1976). Perceptual analysis of motion pattern. Science, 191, 1081-1083. 
Johansson, G. (1950). Configurations in event perception. Uppsala: Almqvist \& Wiksells Boktryckeri AB.

Proffitt, D. R., Cutting J. E., \& Stier, D. M. (1979). Perception of wheel-generated motions. Journal of Experimental Psychology: Human Perception and Performance, 5, 289-302.

Restle, F. (1979). Coding theory of the perception of motion configurations. Psychological Review, 85, 124.

Rock, I., Auster, M., Schiffman, M., \& Wheeler, D. (1980). Induced movement based on the subtraction of motion from the inducing object. Journal of Experimental Psychology: Human Perception and Performance, $6,391-403$.

Rubin, E. (1927). Visuell wahrgenommene wirklich Bewegungen [Visually perceived real motions]. Zeitschrift für Psychologie, 103, 384-392.

Shum, K. H., \& Wolford, G. L. (1983). A quantitative study of perceptual vector analysis. Perception \& Psychophysics, 34, 17-24.

Wallach, H. (1965). Visual perception of motion. In G. Kepes (Ed.), The nature and art of motion (pp. 8597). New York: Braziller.

Wallach, H. (1982). Eye movement and motion. In A. H. Wertheim, W. A. Wagenaar, \& H. W. Leibowitz (Eds.), Tutorials on motion perception (pp. 1-18). New York: Plenum Press.

Wallach, H., \& Becklen, R. (1983). An effect of speed on induced motion. Perception \& Psychophysics, 34. 237-242.

Wallach, H., O'Leary, A., \& McMahon, M. L. (1982). Three stimuli for visual motion perception compared. Perception \& Psychophysics, 32, 1-6.

Received January 30, 1984

Revision received June 15, 1984

\section{Instructions to Authors}

Authors should prepare manuscripts according to the Publication Manual of the American Psychological Association (3rd ed.). All manuscripts must include an abstract of 100-150 words typed on a separate sheet of paper. Typing instructions (all copy must be double-spaced) and instructions on preparing tables, figures, references, metrics, and abstracts appear in the Manual. Also, all manuscripts are subject to editing for sexist language. For further information on content, authors should refer to the editorial in the February 1982 issue of the Journal (Vol. 8, No. 1, pp. 171-172). For information on the other three JEP journals, authors should refer to editorials in those journals.

APA policy prohibits an author from submitting the same manuscript for concurrent consideration by two or more journals. APA policy also prohibits duplicate publication, that is, publication of a manuscript that has already been published in whole or in substantial part in another publication. Authors of manuscripts submitted to APA journals are expected to have available their raw data throughout the editorial review process and for at least 5 years after the date of publication.

Anonymous reviews are optional, and authors who wish anonymous reviews must specifically request them when submitting their manuscripts. Each copy of a manuscript to be anonymously reviewed should include a separate title page with authors' names and affiliations, and these should not appear anywhere else on the manuscript. Footnotes that identify the authors should be typed on a separate page. Authors should make every effort to see that the manuscript itself contains no clues to their identities.

Manuscripts should be submitted in quintuplicate (the original and four photocopies), and all copies should be clear, readable, and on paper of good quality. Authors should keep a copy of the manuscript to guard against loss. Mail manuscripts to the Editor, William Epstein, Department of Psychology, University of Wisconsin, W. J. Brogden Psychology Building, 1202 West Johnson Street, Madison, Wisconsin 53706.

For the other JEP journals, authors should submit manuscripts (in quadruplicate) to one of the editors at the following addresses: Journal of Experimental Psychology: General, Sam Glucksberg, Department of Psychology, Princeton University, Princeton, New Jersey 08544; Journal of Experimental Psychology: Learning, Memory, and Cognition, Henry L. Roediger III, Department of Psychology, Purdue University, West Lafayette, Indiana 47907; and Journal of Experimental Psychology: Animal Behavior Processes, Michael Domjan, Department of Psychology, University of Texas, Austin, Texas 78712. When one of the editors believes a manuscript is clearly more appropriate for an alternative journal of the American Psychological Association, the editor may redirect the manuscript with the approval of the author. 


\title{
Observations
}

\section{Vector Analysis and Process Combinations in Motion Perception: A Reply to Wallach, Becklen, and Nitzberg (1985)}

\author{
Gunnar Johansson \\ Uppsala University, Uppsala, Sweden
}

\begin{abstract}
Wallach, Becklen, and Nitzberg (1985) recently proposed an alternative to the theory of perceptual vector analysis in motion perception. They reported some experimental results, predicted from their own theory but at variance with their predictions from vector theory. Consequently they found their own theoretical approach superior to the vector analysis type of approach. This reply maintains that the authors' conclusion is unwarranted. It also shows that vector theory in a distinct way predicts their experimental results. Furthermore, the epistemological relation between the two theories is clarified, and their mutual predictive power is mentioned.
\end{abstract}

In their article Hans Wallach and his co-workers (Wallach, Becklen, \& Nitzberg, 1985) propose a perceptual theory intended to replace the theory of perceptual vector analysis (called here vector theory for short). As the originator of the vector theory, I want to clarify a programmatic difference between these two theories, correct an interpretation of the experimental results, and discuss some limitations of the proposed theory.

\section{Descriptive and Explanatory Theories}

From an epistemological standpoint, the proposed theory and vector theory represent two different categories. This distinction has not been observed in the target paper (Wallach et al., 1985) but is of decisive importance for the relevance of some of the conclusions drawn by the authors.

Research on visual space and motion perception can belong to two different categories. It can be of the "black box" type and have the character of a mathematical analogue to the visual processing of the optic input. From this approach, many models

Hans Wallach has been a personal friend of mine for a long time, and he stands out as one of my most admired colleagues. Therefore, I could not help feeling uneasy about seriously criticizing an article of which my old friend is the senior author. But I know-and I am sure that Hans Wallach would react in a similar waythat silence is a wrong form of colleagueship and friendship.

Requests for reprints should be sent to Gunnar Johansson, Psykologiska Institutionen, Box 227, S-751 04, Uppsala Universitet, Uppsala, Sweden. for perceptual transfer functions with a good predictive capacity have been developed. Fechner's psychophysics is an early example. The Gestalt laws (Wertheimer, 1923) also represent a theory of this descriptive type, and vector theory belongs to the same category.

A more advanced approach is to start investigating the black box, so to speak, from the inside. In this approach specific sensory processes producing the descriptively established sensory transform are sought. Thus, this is an explanatory approach. Although the descriptive theories with their search for isomorphic relations between stimulus and percept try to answer the question "how," the latter type to answer the question "why." A famous example of advancing from a descriptive to an explanatory theory is found in Köhler's (1947) and Koffka's (1935) magnificent explanation of the Gestalt phenomena in terms of physical processes: physical force fields and electrical activity in the brain. Less spectacular examples can be found, for example, in the classical research on color vision, and, of course, sensory physiology is working with this type of theory. As the title of this article indicates, the theory of Wallach et al. is conceived as a process theory.

There are no reasons for competition or conflict between these two types of theories. They are complementary.

As stated above, in the comparison of their theory with vector theory, Wallach et al. have not taken this important qualitative difference into consideration. In their discussions they ask for specifications of internal sensory processes in vector theory and criticize it for its dependence on an 
"external" analysis. Therefore I must emphasize that vector theory in no way assumes that the vectors should represent specific sensory processes. It is a core assumption of vector theory that the percept is a mathematically correct transformation of the proximal stimulus, and the stimulus events are described in terms of "external," physical processes. The basic methodological principles underlying this theory namely are as follows: (a) Start with highly simplified stimulus events that are perfectly specified in terms of mechanics. (b) Obtain descriptions from the percepts evoked by these stimulus patterns. (c) Transform mathematically the original specification of the stimulus event to the best possible conformity with the percept (Johansson, 1966).

\section{Starting Point of the Wallach et al. Theory}

My next criticism concerns the fact that the authors have not taken the current stage of development of vector theory as their point of departure. Instead they have chosen as their basis two early experiments in Johansson (1950), where the theory of perceptual vector analysis was first formulated. They do not refer to my later research on this theory. However, in Johansson (1950) only a limited and special form of this theory is developed and, in view of my present theoretical position, it is a rather obsolete form. In its initial formulation (Johansson, 1950) vector theory was limited to a two-dimensional and metric form, and the problem of nesting the hierarchical order of relations was not solved in a satisfactory way.

These first investigations of vector theory, however, revealed what was at that time an unexpected side effect: perception of motion in depth. The subjects often reported an alternative way of perceiving the displayed motion patterns. They sometimes saw the relative component as a unitary rigid motion in depth. These observations initiated my still ongoing research on three-dimensional space perception (not limited to vector theory). I found that the initial principle of perceptual vector analysis could be generalized. Today this theory comprehends both static and kinetic three-dimensional space perception, including relations between rigid and nonrigid perception and self-motion perception. Its geometrical structure implies that all veridical "full cue" perceptions of the environment represent a correct vector analysis (cf., e.g., Johansson, 1964, 1966, 1973, 1974a, 1974b, 1975, 1976, 1977, 1978a, 1978b, 1982, 1985).

Given this difference between vector theory at its inception in 1950 and in its present form, it goes without saying that I was both astonished and disappointed to find that Wallach et al. had limited their treatment to an outdated form of vector theory. This limitation implies that their article does not have much relevance for the perceptual research of today. The authors are thirty-five years behind the present front line.

\section{Structure of the Wallach et al. Theory}

In this theory the concept conditions of stimulation plays an important role, and the authors distinguish four such conditions in motion perception. Two of these conditions are of a subjectrelative character and two are object-relative in the authors' terminology. The two subject-relative conditions are ocular pursuit and retinal image displacement; and object-relative ones are orientation change and configurational change, the latter defined as "displacement of the moving object relative to its stationary background (Wallach et al., 1985, p. 93). These conditions of stimulation are assumed to generate three or four different processes. These, however, are not specified in sensory terms, and only the ocular pursuit process seems to be immediately given. Thus, the assumed processes are defined in terms of stimulus conditions. Process combination also plays an important role in the theory. For details see the target paper.

\section{Experiments of Wallach et al. and Their Interpretation}

Their experiments were intended to pit vector theory against the proposed theory. The predictive capacity of the two theories was investigated mainly in the two first experiments. Each experiment had two variants. One variant was a replication of an experiment in Johansson (1950) where two or three dots moved against a homogeneous background. In the second variant a set of stationary dots was superimposed on the diplays from the aforementioned variant. The authors find that both theories predict the same vector analysis effect in the variant representing the direct replication of the Johansson experiments.

Introducing stationary dots in the second variant implies a configurational change as defined by the authors. Therefore, the authors correctly state that their theory predicts that in this case the moving dots will be seen as moving relative to the stationary part of the pattern, thus creating a veridical perception. This was indeed the experimental result.

When it is a question of prediction from vector theory, the authors state this theory should expect the same result from this variant as from the original display. They offer no substantial argument for this; they just state that "there is no reason 
why the presence of stationary dots should prevent such an extraction [of common components]" (p. 99). From this interpretation they conclude that vector theory has only a limited and superficial validity.

This interpretation of vector theory is very puzzling. Analysis of configurational change (including stationary configurational elements) forms the methodological basis for vector theory. A general result from all the experiments in Johansson (1950) as well as in later publications is that change in the spatiotemporal relations between displayed elements regularly results in changes in the perceptual outcome. Thus, from this background the predictions should be that introducing new elements in surround-stationary or notmust bring about a perceptual change.

The difference between the prediction advanced by Wallach et al. and my own is, of course, of a crucial importance for the relevance of Wallach's conclusions about vector theory. Therefore, I find it necessary to present a detailed prediction from an argumentation built on an explicit theoretical basis. This basis is given in the following brief excursus, mainly on the principle of relativity in vector theory.

\section{Basic Structure of Vector Theory}

1. The head of an active perceiver is very seldom stationary. At the nodal point of the eye, head movements relative to the environment bring about continuous changes of visual direction to the distal light-reflecting points. Therefore, in accordance with Gibson (1966), the stimulus is specified as an optic flow on the retina, generated by these angular changes of direction. (This implies that effects of eye movements are not included in the model.)

2. Some form of structure in the light reflected into the eye is a necessary condition for an optic flow. Without an optic flow, an empty threedimensional space is perceived.

3. The optic flow generated by structures in three-dimensional space is analyzed in terms of invariant relations under perspective transformation. Such invariant relations in the optic flow are treated as mathematical analogues to perception of rigid motion. The head-motion generated flow in a rigid environment specifies this as being rigid (Gibson, 1950, 1957).

4. The local visual flow generated by a rigid object (or an experimental element) moving against a static background can be specified as a vector sum of the background flow generated by selfmotion and the flow component generated by the object's motion relative to the background. Projections from motion relative to moving objects can be described in an analogous way, and thus hierarchical chains or trees of relative motions are obtained. According to vector theory, the rules that govern the visual system's abstraction of information from the total optic flow correspond to the rules for description of the flow in terms of relative motions.

5. It follows from the above points that vector theory can describe and correctly predict all veridical perception. It supposes that the visual system works in a mathematically correct way, and it affords principles for a mathematical transform of the proximal stimulus that is equivalent to the percept. Experimentally, perceptual vector analysis, therefore, can be demonstrated only by a deliberate reduction of information in the optic flow, bringing about a nonveridical perception. The most dramatic and convincing demonstrations so far of the validity of this theory are found in the great number of studies and demonstrations on the biological motion effect, started by Johansson $(1973,1976)$.

6. Under conditions with impoverished optic information and conflicting motion relations, the visual system often responds with a compromise. Gogel (1974) showed that the perceived direction of a motion track is a function of the relative proximity to the rivaling motions. Compare Hochberg and Fallon (1976) and the vector synthesis in Johansson (1950). The target article also nicely demonstrates this effect.

\section{Rationale for My Own Prediction}

In the experiments in Johansson (1950), revealing the principle of perceptual vector analysis, the relation between a structured background and the moving elements was efficiently reduced. In this way a component in the veridical perception was removed. The result was nonveridical percepts, which could be varied experimentally.

With this background in mind and with special reference to Point 4 above, the prediction from vector theory about what will happen in the experiments of Wallach et al. must be perfectly clear. The vector analysis effect studied in the replications of original experiments was the result of an approximate elimination of information about the background component from the display. Introducing in this display the removed information about a stationary framework, as in the authors' stationary-dot variant, must, in accordance with the theory, result in seeing the moving dots as moving relative to this framework, that is, in veridical perception. Thus, the obtained experimental results are predicted. 
Shortcomings of the Wallach et al. Theory in Explaining Later Experiments on Vector Theory

The discussion so far has made clear that Wallach et al.'s process theory and vector theory, contrary to the authors' statement, yield concordant predictions about the outcome from the experiments discussed; therefore, the authors' allegation of superiority of their own theory is unwarranted. As mentioned previously, however, this must be regarded as being of rather limited interest. A more relevant question is whether this theory can also explain later experimental results from vector theory. Thus I will point out some significant shortcomings in this respect.

\section{The Wallach et al. Theory Is Restricted to Two-Dimensional Perception}

Hans Wallach has in the past made outstanding contributions to the understanding of perception of motion in depth. Like the vector theory in Johansson (1950), however, his present process theory can analyze only two-dimensional aspects of visual motion perception. Before this theory can be accepted as a useful contribution to the visual research of today, it must also be capable of handling three-dimensional motion perception in specified process terms.

\section{The Wallach et al. Theory Is Unable To Explain Complex Hierarchies}

As I understand it, the Wallach et al. theory can deal only with hierarchies with, at most, one subject-relative motion, one translatory motion, and one rotary motion. This implies that it is not capable of handling some of the most important results from the later experiments on vector theory. This theory has received its most convincing support from experiments on complicated hierarchies of relative motions. The analyses of biological motion effects already referred to (Johansson, 1973, 1976) afford the most advanced examples so far. These displays are vectorially analyzed as branching trees consisting of a very great number of hierarchical relations. Initially, this type of complicated stimulus pattern was constructed by predictions from vector theory.

\section{Subjective-Relative Processing Is Not a Necessary Condition for Perceptual Vector Analysis}

In the theory of Wallach et al., the existence of a subject-relative motion stands out as a necessary condition for explaining vector analysis. In several experiments on perceptual vector analysis, however, this effect has been demonstrated from displays lacking a component of subject-relative motion in the sense that Wallach et al. use the term. The experiments of Johansson (1964) afford good examples (also shown in the film by Maas, 1971). In these experiments, displays in the form of surfaces changing shape in a symmetrical way evoked the perception of sagittal motion in depth and a simultaneous rotation or, alternatively, a form change of the surface. The stimulus conditions for perceiving sagittal motion have no subjectrelative motion. Another example can be found in Johansson (1976) and in Maas (1971), where frontoparallel biological motion is perceived without any subject-relative displacement of the pattern. Thus such a process is not a necessary condition for perceptual vector analysis.

These three considerations clearly show that the Wallach et al. theory in its present form, especially with regard to its linkage to subject-relative motion and two-dimensional perception, cannot explain the effects of perceptual vector analysis and act as a substitute for this theory.

\section{References}

Gibson, J. (1950). The perception of the visual world. Boston: Houghton Mifflin.

Gibson, J. J. (1957). Optical motions and transformations as stimuli for visual perception. Psychological Review, 64, 288-295.

Gibson, J. J. (1966). The senses considered as perceptual systems. New York: Houghton Mifflin.

Gogel, W. C. (1974). Relative motion and the adjacency principle. Quarterly Journal of Experimental Psychology, 14, 425-437.

Hochberg, J., \& Fallon, P. (1976). Perceptual analysis of moving patterns. Science, 194, 1081-1083.

Johansson, G. (1950). Configurations in event perception: Uppsala, Sweden: Almqvist \& Wiksells.

Johansson, G. (1964). Perception of motion and changing form. Scandinavian Journal of Psychology. 5, 181208.

Johansson, G. (1966). Geschehenswahrnemung [Event perception]. In W. Metzger \& H. Erke (Eds.), Handbuch der Psychologie [Handbook of psychology] (Vol. 1, pp. 745-775). Göttingen, West Germany: Verlag für Psychologie.

Johansson, G. (1973). Visual perception of biological motion and a model for its analysis. Perception \& Psychophysics, 14, 201-211.

Johansson, G. (1974a). Projective transformations as determining visual space perception. In R. B. MacLeod \& H. L. Pick, Jr. (Eds.), Perception: Essays in honor of J. J. Gibson (pp. 117-138). Ithaca, NY: Cornell University Press.

Johansson, G. (1974b). Vector analysis in visual perception of rolling motion: A quantitative approach. Psychologische Forschung. 36, 311-319.

Johansson, G. (1975). Visual motion perception. Scientific American, 232, 76-88.

Johansson, G. (1976). Spatio-temporal differentiation and integration in visual motion perception. Psychological Research, 38, 379-393. 
Johansson, G. (1977). Spatial constancy and motion in visual perception. In W. Epstein (Ed.), Stability and constancy in visual perception (pp. 375-419). New York: Wiley.

Johansson, G. (1978a). About the geometry underlying spontaneous visual decoding of the optical message. In E. L. J. Leuwenberg \& H. F. J. Buffart (Eds.), Formal theories of visual perception (pp. 265-276). New York: Wiley.

Johansson, G. (1978b). Visual event perception. In R. Held, H. W. Leibowitz, \& H.-L. Teuber (Eds.), Handbook of sensory physiology (Vol. 8, pp. 675-711). Berlin: Springer-Verlag.

Johansson, G. (1982). Visual space through motion. In A. H. Wertheim, W. A. Wagenaar, \& H. W. Leibowitz (Eds.), Tutorials on motion perception (pp. 19-39). New York: Plenum Press.

Johansson, G. (1985). About visual event perception. In
W. H. Warren \& R. E. Shaw (Eds.), Persistence and change: Proceedings of the First International Conference on Event Perception. Hillsdale, NJ: Erlbaum.

Koffka, K. (1935). Principles of Gestalt psychology, New York: Harcourt, Brace.

Köhler, W. (1947). Gestalt psychology. New York: Liveright. Maas, J. B. (Producer). (1971). Motion perception [Film, Parts 1 \& 2]. Boston: Houghton Mifflin.

Wallach, H., Becklen, R., \& Nitzberg, D. (1985). Vector analysis and process combination in motion perception. Journal of Experimental Psychology: Human Perception and Performance, 11, 93-102.

Wertheimer, M. (1923). Untersuchungen zur Lehre von der Gestalt [Investigations of Gestalt theory]. Psychologische Forschung, 4, 301-350.

Received October 24, 1984 


\title{
Response to Gunnar Johansson's Critical Commentary
}

\author{
Hans Wallach \\ Swarthmore College
}

\author{
Robert Becklen \\ Sarah Lawrence College
}

\begin{abstract}
Vector theory seems to furnish adequate descriptions of the motions that are perceived in Johansson's 1950 displays, motions that are perceived when objects move while the visual field is being displaced relative to the head during the head's turning or nodding, or motions that are perceived when they move where the visual field is expanding during forward locomotion. But in the first two cases a better understanding is reached when one considers the stimulus conditions that operate: The simultaneous motions that are often perceived in Johansson's displays are the result of two kinds of stimulation that both represent the display motion and directly result in different perceived motions. When objects move during head movements, compensatory eye movements cause the visual field to remain stationary on the retinas, and retinal image displacements correctly represent object motions. Only perception of objective motions that take place during forward locomotion in shifting parts of the expanding visual field requires explanations provided by vector theory. Organic motions are not considered.
\end{abstract}

The correct description of a set of phenomena can be a major contribution. That is true of the way Johansson described the perceived motions caused by the displays that he originated more than 30 years ago. Johansson recognized that these motions result from vector analysis; whether the analysis is performed by the perceptual processes as Johansson conceived of it in 1950 or whether the nature of physical motion makes the analysis possible as we (Wallach, Becklen, \& Nitzberg, 1985 ) see it is a minor matter. Moreover, it is no issue at all in Johansson's contemporary vector theory, which is meant to be purely descriptive.

Johansson (1985) compares our explanation of the motions that are seen when his displays are viewed to Köhler's "magnificent" but, in our view, untenable physicalistic theory of cortical figure processes or to theories of sensory physiology, but this comparison requires a correction. The different stimulus conditions that we believe are responsible for the different simultaneously perceived motions are either contained in the projections on the retinas or are directly caused by them, or they consist in tracking eye movements, but assumptions about the physiological processes they evoke are not part of the explanation; the mere existence of these stimulus conditions suffices. Furthermore, the three stimulus conditions are no mere postulates. They are experimentally established facts. Their relative effectiveness has been measured (Wallach, O'Leary, \& McMahon, 1982); the ac-

Requests for reprints should be sent to Hans Wallach, Department of Psychology, Swarthmore College, Swarthmore, Pennsylvania 19081. curacies of the shapes of the perceived motion paths that were provided by each stimulus condition have been studied and have been compared with each other (Wallach, O'Leary, \& McMahon, in preparation), and, in the case of two of them, the perceived motions they evoke have been altered by adaptation (Bacon \& Wallach, 1982; Wallach, Bacon, \& Schulman, 1978).

Our experiments with Johansson's displays were part of ongoing research concerned with problems that arise in motion perception when two of the redundant stimuli operate simultaneously, as they usually do (Wallach, 1984), and were conceived in this context. We hope that our article is a successful attempt to explain, with the help of current knowledge in motion perception, those instances of vector analysis that take place when Johansson's displays of 1950 are observed. We do not address Johansson's general vector theory for two reasons: We have done no experiments with biological motions, and we do not believe in the pervasive presence of vector analysis that the theory envisions.

The last point requires an explanation. Whenever the head is turned or nodded, the visual environment is continuously displaced relative to the head. This flow encompasses everything that is seen. When an object moves during a head movement, its displacement combines kinematically with this flow, and perceiving the motion of the object correctly requires a vector analysis of the kinematic resultant of the object's displacement and the general flow. This vector analysis is the result of the hierarchical structure of motion perception, which treats the general flow and the displacement of the moving object differently. The latter's perceived motion results from its displace- 
ment relative to the flowing background, and this makes it veridical. This is Johansson's account. It is correct if it is taken as a mathematical description of what must occur when the motion of an object is perceived correctly during a head movement, but when we look at the details of how the visual system deals with the relative environmental flow caused by a head movement, we find that vector analysis is rarely needed. During head movements, the eyes make compensatory movements so that the retinal projection of the environment remains stationary on the retinas. These compensatory eye movements have been called a reflex, because they take place even in the dark when nothing is visible on which the eyes can fasten. If these compensatory eye movements were not taking place and the eyes did not move in the head, the visual field would shift on the retinas and would be blurred during each head movement. This blurring can be easily observed by fixing the eyes on a point straight ahead that is made to move with the head, for example, the tip of a wire whose other end is wound around one's head. When moving the head causes this point and, along with it, the gaze to shift relative to the visual environment, the latter appears blurred. Compensatory eye movements can, however, be altered by adaptation (Wallach \& Bacon, 1977, pp. 233-234). Because the compensatory eye movements keep the projection of the environment stationary on the retinas, objects that move during head movements are given on the retinas by images whose displacements represent the motions of the object correctly, and no vector analysis is needed.

Eye movements cannot compensate when forward locomotion causes continuous expansion of the subject's whole visual field, and large parts of the environment are given on the retinas in a radial flow pattern. This may be an instance where hierarchical chains of relative motions do operate and cause correct perception of motions that are given only as components of resultant motions because the moving objects participate in radial flow.

Apart from the result of forward locomotion, continuous displacement of the projection of the environment on the retina occurs only when the eyes track a moving object. It is noteworthy that under these conditions a second object's motion that differs in direction from the tracked motion is not correctly perceived; the vector analysis that would be needed here does not take place (Becklen, Wallach, \& Nitzberg, 1984). No stationary environment was visible in these experiments, but that is also the case in Johansson's 1950 displays, if vector analysis is to be demonstrated.

\section{References}

Bacon, J., \& Wallach, H. (1982). Adaptation in motion perception: Alteration of motion evoked by ocular pursuit. Perception \& Psychophysics, 31, 251-255.

Becklen, R., Wallach, H., \& Nitzberg, D. (1984). A limitation of position constancy. Journal of Experimental Psychology: Human Perception and Performance, 10, 713-723.

Johansson, G. (1950). Configurations in event perception. Uppsala, Sweden: Almqvist \& Wiksells.

Johansson, G. (1985). Vector analysis and process combination in motion perception: A Reply to Wallach, Becklen, and Nitzberg (1985). Journal of Experimental Psychology: Human Perception and Performance, 11. 367-371.

Wallach, H. (1984). Learned stimulation in space and motion perception. American Psychologist, 40, 399404.

Wallach, H., \& Bacon, J. (1977). Two kinds of adaptation in the constancy of visual direction and their different effects on the perception of shape and visual direction. Perception \& Psychophysics, 21, 227-241.

Wallach, H., Bacon, J., \& Schulman, P. (1978). Adaptation in motion perception: Alterations of induced motion. Perception \& Psychophysics, 24, 509-514.

Wallach, H., Becklen, R., \& Nitzberg, D. (1985). Vector analysis and process combination in motion perception. Journal of Experimental Psychology: Human Perception and Performance, 11, 93-102.

Wallach, H., O'Leary, A., \& McMahon, M. L. (1982). Three stimuli for visual motion perception compared. Perception \& Psychophysics, 32, 1-6.

Wallach, H., O'Leary, A., \& McMahon, M. L. (1983). Perceived motion paths resulting from different stimuli. Manuscript in preparation.

Received December 5, 1984 